\title{
Predictive factors associated with relapse of stage II/III colon cancer treated with peroral anti-cancer agents in the adjuvant setting
}

\author{
YUSUKE MIZUUCHI $^{1,2}$, YOSHITAKA TANABE ${ }^{1}$, MASAFUMI SADA $^{1,2}$, YOSHIKI KITAURA $^{1}$, \\ SHUNTARO NAGAI $^{2}$, YUSUKE WATANABE ${ }^{1,2}$, SADAFUMI TAMIYA $^{3}$, KINUKO NAGAYOSHI ${ }^{2}$, \\ KENOKI OHUCHIDA $^{2}$, TORU NAKANO ${ }^{1}$ and MASAFUMI NAKAMURA ${ }^{2}$
}

\author{
${ }^{1}$ Department of Surgery, Kitakyushu Municipal Medical Center, Kitakyushu, Fukuoka 802-8561; ${ }^{2}$ Department of \\ Surgery and Oncology, Graduate School of Medical Sciences, Kyushu University, Higashi, Fukuoka 812-8582; \\ ${ }^{3}$ Department of Diagnostic Pathology, Kitakyushu Municipal Medical Center, Kitakyushu, Fukuoka 802-8561, Japan
}

Received October 11, 2020; Accepted February 26, 2021

DOI: $10.3892 / \mathrm{mco} .2021 .2284$

\begin{abstract}
Postoperative adjuvant chemotherapy for patients with stage III colon cancer (CC) is regarded as the standard treatment worldwide for outcome improvement and relapse prevention. Similarly, high-risk stage II CC requires adjuvant chemotherapy because of its high recurrence rate. Previous randomized controlled trials showed that oxaliplatin (OX), in addition to fluorinated pyrimidine-based therapy for patients with stage II/III CC, significantly improves cancer survival but it remains controversial as to which patient groups should receive OX-containing regimens. Among 1,150 consecutive patients who underwent curative resection for stage II/III CC between 2009 and 2016 at two tertiary hospitals, 349 patients treated with only peroral (PO) fluorinated pyrimidine-based chemotherapy and 149 patients who received fluorinated pyrimidine-based chemotherapy with OX as adjuvant chemotherapy were retrospectively reviewed. The primary outcome was recurrence-free survival (RFS). Clinicopathological factors were more advanced in patients treated with $\mathrm{OX}$ than in patients treated only with PO fluorinated pyrimidine agents. Multivariate analysis for 5-year RFS showed that T4 [hazard ratio (HR), 2.947; $\mathrm{P}=0.0001], \mathrm{N} 2(\mathrm{HR}, 2.704 ; \mathrm{P}=0.0075)$, vessel or lymphatic invasion $(\mathrm{HR}, 1.675 ; \mathrm{P}=0.0437)$ and high cancer antigen (CA)19-9 (HR 3.367, $\mathrm{P}=0.0002)$ levels were independent risk factors of cancer relapse. Propensity score
\end{abstract}

Correspondence to: Dr Yusuke Mizuuchi, Department of Surgery, Kitakyushu Municipal Medical Center, 2-1-1 Bashaku, Kokurakita, Kitakyushu, Fukuoka 802-8561, Japan

E-mail: mizuy@med.kyushu-u.ac.jp

Abbreviations: CC, colon cancer; PO, peroral; RFS, recurrence-free survival; OX, oxaliplatin; CEA, carcinoembryonic antigen

Key words: colon cancer, adjuvant chemotherapy, peroral fluorinated pyrimidine agent, oxaliplatin, cancer antigen 19-9 matching analysis was performed to match clinicopathological differences between the PO and OX groups. After matching, subgroup analysis of the patients showed that greater effects of OX on cancer survival were observed in patients in the OX group with high CA19-9 levels and tended to be associated with T4 and N2 compared with the PO group. Thus, OX-containing regimens should be recommended for patients with $\mathrm{CC}$ with these factors in an adjuvant setting.

\section{Introduction}

Colon cancer (CC) is the third highest cause of cancer-associated mortality in the United States (1). In Japan, CC is the leading cause of mortality in women and the third highest cause in men. A total of 101,952 patients were newly diagnosed with CC in 2017 and 35,414 patients died due to CC in 2018 (2). Surgery is the most suitable treatment for patients with resectable $\mathrm{CC}$ and the number of laparoscopic surgeries performed in Japan is increasing (3). In advanced $\mathrm{CC}$ with distant metastases, prolonged survival be achieved by multidisciplinary therapy such as surgery, chemotherapy, immunotherapy and radiation, but patients still have a poor prognosis; the 5-year overall survival rate is $16.7 \%$ in Japan (4). Because recurrent $\mathrm{CC}$ also has a poor prognosis, recurrence prevention contributes to extending survival. Postoperative adjuvant chemotherapy aims to decrease recurrence in patients with stage II/III disease following R0 surgery.

Oxaliplatin (OX), a therapeutic in the platinum-based antineoplastic family, acts by blocking DNA replication and is a key drug together with fluorinated pyrimidine. Based on the results of Multicenter International Study of OX/5-FU-LV in the Adjuvant Treatment of CC (MOSAIC), National Surgical Adjuvant Breast and Bowel Project (NSABP) and capecitabine + OX adjuvant (XELOXA) trials (5-8), OX-containing regimens such as folinic acid + fluorouracil $(\mathrm{FU})+\mathrm{OX}(\mathrm{FOLFOX})$, infused 5-FU + leucovorin + OX (FLOX) and peroral capecitabine + infused OX (CapOX) are standard therapies for postoperative adjuvant chemotherapy for patients with $\mathrm{CC}$. However, in $\mathrm{OX}$ induction, persistent 
OX-induced peripheral neuropathy is often an adverse effect and a number of patients are concerned about this refractory symptom (9).

While OX-containing regimens are a worldwide standard in the adjuvant setting for postoperative patients with $\mathrm{CC}, \mathrm{PO}$ fluorinated pyrimidine-based therapies, such as uracil-tegafur/ leucovorin (UFT/LV), tegafur/gimeracil/oteracil (S-1) and capecitabine are commonly used, especially in Japan for patients with CC with a relatively low risk of recurrence (5-8). Treatment with such PO fluorinated pyrimidine agents is also standard, based on the results of NSABP, Japan Clinical Oncology Group, Xeloda in Adjuvant CC Therapy (ACT), ACT Stage III CC and Stage II/III Rectal Cancer trials (10-14).

$\mathrm{T} 4$, N2, high levels of tumor markers, including carcinoembryonic antigen (CEA) and cancer antigen (CA)19-9, and lymphatic or vessel permeation have been reported as high-risk factors for CC recurrence (15-18); however, a subgroup analysis of previous randomized trials did not show superiority of OX-containing regimens in patients with these high-risk factors $(5,6,8)$. Thus, it remains controversial as to which patients should be treated with OX-containing regimens. The present study aimed to identify patients with high relapse rates by analyzing clinicopathological factors among those treated with PO fluorinated pyrimidine agents without OX and used propensity score matching analysis to determine whether patients with certain characteristics should receive more effective chemotherapeutic agent-containing regimens, such as those that incorporate OX.

\section{Materials and methods}

Patients and data collection. The inclusion criteria were consecutive patients with CC with curative resection who underwent adjuvant chemotherapy and were histologically diagnosed as stage II/III colon adenocarcinoma at two tertiary medical institutions (Kitakyushu Municipal Medical Center, Kitakyushu, and the Department of Surgery and Oncology at Kyushu University, Higaki, Japan) between September 2009 and March 2016. Patients with two or more cancer lesions or who had been treated with preoperative adjuvant therapy were excluded. Medical records of 1,065 patients with stage II/III CC were retrospectively reviewed and 501 patients with postoperative adjuvant chemotherapy were identified. Three patients treated with irinotecan-containing regimens were excluded. Finally, a total of 349 and 149 patients treated with PO and OX-containing regimens, respectively, were enrolled for subsequent analysis. The median observation period was 1,689 days. The informed consent requirement was waived because of the retrospective nature of the study, in which patient data was kept confidential. All procedures conformed to the ethical guidelines of the Japanese Government and the Declaration of Helsinki. This retrospective study was approved by the ethics committee of Kitakyushu Municipal Medical Center (approval no. 201801055).

Clinicopathological factors. Preoperative variables were institution, sex, age, tumor location, preoperative bowel obstruction and preoperative serum levels of CEA and CA19-9. The intraand postoperative variables were surgical procedure (open or laparoscopic), blood loss volume, operating time, number of harvested lymph nodes, hospital stay length and morbidity. Histological variables were tumor differentiation, $\mathrm{T}$ and $\mathrm{N}$ stage and lymphatic or vessel invasion. Tumor location was defined as follows: Right colon, including cecum, ascending and transverse colon; and left colon, including descending and sigmoid colon and rectosigmoid junction. The pathological tumor stage was defined according to the Union for International Cancer Control (UICC) TNM classification (19). Tumor differentiation was defined as well-, moderately and poorly differentiated (20). Postoperative complications were evaluated using the Clavien-Dindo classification (21) as follows: Grade I, deviation from the normal course without need for treatment; grade II, complication requiring pharmacological treatment; grade III, complication requiring surgical, endoscopic or radiological intervention; grade IV, life-threatening complication requiring intensive care unit management; and grade $\mathrm{V}$, patient mortality. In the present study, complications greater than grade II were defined as postoperative complications. Postoperative mortality indicated 30-day or in-hospital deaths.

Adjuvant chemotherapy regimens. Postoperative adjuvant chemotherapy, regimens and dosages were determined by individual attending physicians, referring to the multi-disciplinary cancer board. Patients who received adjuvant chemotherapy for stage II cancer without high risk factors for recurrence (T4, lymphatic or vascular invasion, preoperative bowel obstruction and high histological grade) were excluded from the present study. All high-risk stage II and stage III patients were considered suitable for adjuvant chemotherapy, except for those intolerant to chemotherapy and those who did not consent to chemotherapy. In most cases, postoperative adjuvant chemotherapy was administered to patients with stage III colorectal cancer (CRC), but it was not recommended for all patients with stage II CRC. OX-containing regimens were recommended primarily for patients with $\geq 4$ lymph node metastases (N2), tumor-invaded adjacent organs or perforated visceral peritoneum (T4), but OX could be initiated at other stages following a decision by either the patient or the treating physician referring to the multi-disciplinary cancer board.

Statistical analysis. The association between two variables was analyzed using chi-squared or Fisher's exact test as appropriate. For the survival analysis, recurrence-free survival (RFS) was adopted as the primary endpoint. Recurrence data included the presence and date of recurrence and the last follow-up date in patients without recurrence. The last follow-up date was December 2020. The survival analysis was performed using Kaplan-Meier analysis and the curves were compared using the log-rank test. Continuous variables were expressed as the median and the range was assessed using Mann-Whitney U test. The cutoff of the tumor marker was determined according to the upper normal limit at Kitakyushu Municipal Medical Center and Kyushu University Hospital. Multivariate survival analysis was evaluated by Cox's proportional hazard model. In order to minimize the impact of selection bias and other confounding factors, propensity score-matched analysis was performed as previously described (22). Propensity scores were generated using clinicopathological characteristics including institution, age, sex, tumor location and differentiation, 
Table I. Chemotherapy regimens administered to patients with colorectal cancer.

A, Peroral group

\begin{tabular}{lr}
\hline Regimen & $\mathrm{n}$ \\
\hline Cap & 202 \\
UFT/LV & 85 \\
S-1 & 29 \\
UFT & 31 \\
5'-DFUR & 2 \\
Total & 349 \\
\hline
\end{tabular}

\section{$\mathrm{B}, \mathrm{OX}$ group}

\begin{tabular}{lr}
\hline Regimen & $\mathrm{n}$ \\
CapOX & 93 \\
mFOLFOX6 & 53 \\
SOX & 3 \\
Total & 149 \\
\hline
\end{tabular}

Cap, Capecitabine; UFT/LV, uracil-tegafur/leucovorin; S-1, tegafur/gimeracil/oteracil; 5'-DFUR, 5'-deoxy-5-fluorouridine; OX, oxaliplatin; mFOLFOX6, modified folinic acid + fluorouracil + OX 6; SOX, S-1 + OX.

pathological T and $\mathrm{N}$ stage and lymphatic or vessel invasion. Propensity scores were matched using a caliper width of 0.05 multiplied by the standard deviation of values calculated by a logistic regression model. Each patient with an OX-containing regimen was matched to a patient with a PO regimen using a one-to-one nearest neighbor-matching algorithm without replacement. Following matching, there were 105 patients in each group. All statistical analyses were performed using JMP 15 software (SAS Institute, Inc.). $\mathrm{P}<0.05$ was considered to indicate a statistically significant difference.

\section{Results}

Patient characteristics. Fig. 1 shows the distribution of patients. Among all patients who underwent curative resection and were diagnosed with colon adenocarcinoma, 85 patients who either had $\geq 2$ cancer occurrences or underwent preoperative therapy were excluded. A total of 564 patients received no postoperative adjuvant chemotherapy, 149 patients were treated with OX-containing regimens and 349 patients were treated with PO fluorinated pyrimidine agent. In addition, we also excluded three patients who received irinotecan-containing regimens (folinic acid $+\mathrm{FU}+$ irinotecan and irinotecan $+\mathrm{S}-1$ )

Table I details the chemotherapy regimens of the PO and OX groups. Patients in the PO group were administered sole UFT and 5'-deoxy-5-fluorouridine regimens at a relatively early stage.

A number of patients without adjuvant chemotherapy were T3N0 patients without other high-risk factors for recurrence. According to UICC TNM stage, among the stage II patients, almost all patients who underwent adjuvant

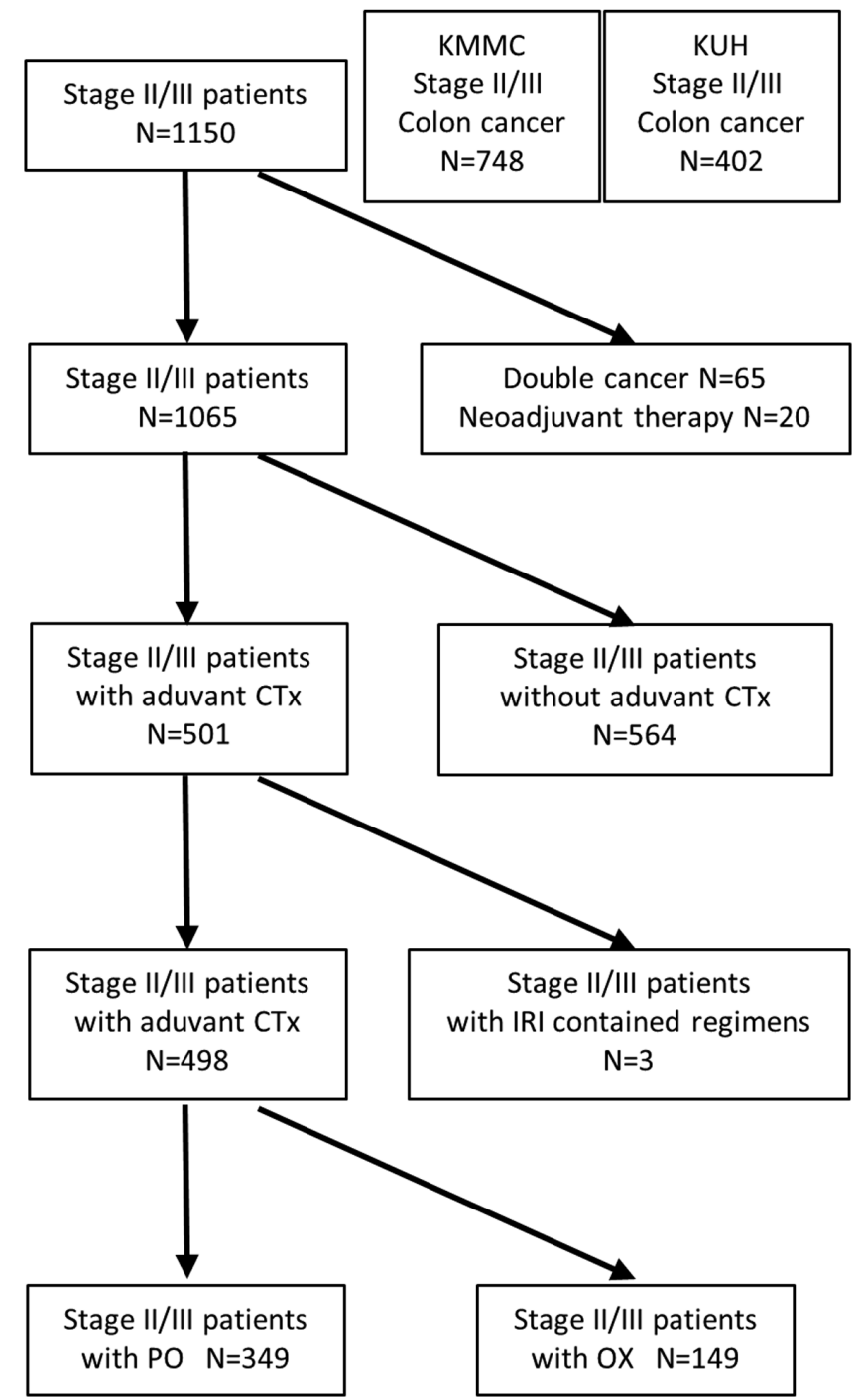

Figure 1. Flow chart showing the distribution of patients. KMMC, Kitakyushu Municipal Medical Center; KU, Kyushu University Hospital; CTx, chemotherapy; IRI, irinotecan; PO, peroral; OX, oxaliplatin.

chemotherapy were high-risk stage II (high histological grade, T4 and lymphatic or vessel invasion). There were 20 patients in the OX group and 103 patients in the PO group, which indicates that a large number of regimens for patients with stage II CRC in the adjuvant setting were PO regimens without $\mathrm{OX}$. Among the stage III patients, there were 129 patients in the OX group and 246 patients in the PO group. Two patients without adjuvant chemotherapy died postoperatively; one patient with perforating peritonitis and sepsis due to $\mathrm{CC}$ died of multiple organ failure 2 days after emergency surgery; another patient with arteriosclerosis obliterans and poorly controlled diabetes died of multiple organ failure due to anastomotic leakage 4 months after elective surgery. No chemotherapy-associated mortality was identified in the PO and OX groups.

Table II details the characteristics of patients in the OX and $\mathrm{PO}$ groups. Compared with the PO group, the age of the OX group patients was younger $(\mathrm{P}>0.001)$, poorly differentiated adenocarcinoma was more frequently observed $(\mathrm{P}=0.023)$ and $\mathrm{T}(\mathrm{P}=0.033), \mathrm{N}(\mathrm{P}>0.001)$, and UICC TNM 
Table II. Characteristics of patients with colon cancer treated with adjuvant chemotherapy.

\begin{tabular}{|c|c|c|c|}
\hline Characteristic & OX group & PO group & P-value \\
\hline $\mathrm{N}$ & 149 & 349 & \\
\hline Male/female & $77 / 72$ & $142 / 207$ & $0.024^{\mathrm{a}}$ \\
\hline Mean age, years & $61.8(30.0-84.0)$ & $66.7(31.0-89.0)$ & $>0.001^{\mathrm{a}}$ \\
\hline Institution (KMMC/KU) & $78 / 71$ & $239 / 110$ & $0.007^{\mathrm{a}}$ \\
\hline Tumor location & & & 0.465 \\
\hline Right & $55(36.9 \%)$ & $141(40.4 \%)$ & \\
\hline Left & $92(63.1 \%)$ & $208(59.6 \%)$ & \\
\hline \multicolumn{4}{|l|}{ Tumor marker } \\
\hline Median CEA & $3.6(0.6-168.0)$ & $3.0(0.4-262.1)$ & 0.529 \\
\hline Median CA19-9 & $9.0(1.5-2351.8)$ & $8.0(1.8-592.2)$ & 0.342 \\
\hline Bowel obstruction & $22(14.8 \%)$ & $37(10.6 \%)$ & 0.196 \\
\hline Surgical procedure & & & 0.388 \\
\hline Laparoscopic & $132(88.6 \%)$ & $318(91.1 \%)$ & \\
\hline Open & $17(11.4 \%)$ & $31(8.9 \%)$ & \\
\hline Median blood loss volume, $\mathrm{ml}$ & $30(2-2965)$ & $30(3-11010)$ & 0.516 \\
\hline Median operating time, min & $251(88-752)$ & $241(90-594)$ & 0.114 \\
\hline Number of lymph nodes & $25(3-97)$ & $21(2-112)$ & \\
\hline Hospital stay, days & $13(5-137)$ & $13(7-125)$ & 0.738 \\
\hline Postoperative complications & $25(16.8 \%)$ & $50(14.3 \%)$ & 0.487 \\
\hline Tumor differentiation & & & $0.023^{\mathrm{a}}$ \\
\hline Well & $70(47.0 \%)$ & $200(57.3 \%)$ & \\
\hline Moderate & $56(37.6 \%)$ & $121(34.7 \%)$ & \\
\hline Poor & $23(15.4 \%)$ & $28(8.0 \%)$ & \\
\hline T stage & & & $0.033^{\mathrm{a}}$ \\
\hline $\mathrm{T} 1$ & $3(2.0 \%)$ & $16(4.6 \%)$ & \\
\hline $\mathrm{T} 2$ & $17(11.4 \%)$ & $30(8.6 \%)$ & \\
\hline $\mathrm{T} 3$ & $97(65.1 \%)$ & $258(73.9 \%)$ & \\
\hline $\mathrm{T} 4$ & $32(21.5 \%)$ & $45(12.9 \%)$ & \\
\hline $\mathrm{N}$ stage & & & $>0.001^{\mathrm{a}}$ \\
\hline N0 & $20(13.4 \%)$ & $103(29.5 \%)$ & \\
\hline $\mathrm{N} 1$ & $72(48.3 \%)$ & $226(64.8 \%)$ & \\
\hline $\mathrm{N} 2$ & $57(38.3 \%)$ & $20(5.7 \%)$ & \\
\hline Vessel or lymphatic invasion & $81(54.4 \%)$ & $150(43.0 \%)$ & $0.019^{\mathrm{a}}$ \\
\hline TNM stage & & & $>0.001^{\mathrm{a}}$ \\
\hline II & $20(13.4 \%)$ & $103(29.5 \%)$ & \\
\hline III & $129(86.6 \%)$ & $180(70.5 \%)$ & \\
\hline
\end{tabular}

${ }^{\mathrm{a}} \mathrm{P}<0.05$. CEA, carcinoembryonic antigen; CA, cancer antigen; OX, oxaliplatin; PO, peroral; KMMC, Kitakyushu Municipal Medical Center; KU, Kyushu University Hospital.

stage $(\mathrm{P}>0.0001)$ were advanced. Vessel or lymphatic invasion was also observed more frequently $(\mathrm{P}=0.019)$. The proportion of patients in the OX group differed between the two institutions $(\mathrm{P}=0.007)$, reflecting the policy of each institution regarding the use of $\mathrm{OX}$-containing regimens. Tumor location, tumor markers, preoperative bowel obstruction, surgical procedure, blood loss volume, operating time, number of harvested lymph nodes, postoperative hospital stay and morbidity were not different between the two groups. These results suggested physician selection bias as a large proportion of $\mathrm{N} 1$ patients were treated with $\mathrm{PO}$ chemotherapy, while patients with more aggressive N2 almost exclusively received OX-containing regimens.

Comparison of RFS between patients treated with $P O$ and OX-containing regimens. In the PO group, 66 of 349 (18.9\%) patients had a recurrence of $\mathrm{CC}$, with initial relapse in the liver $(n=28)$ or lung $(n=19)$, tumor dissemination $(n=12)$ and lymph node $(n=15)$, local $(n=3)$ or bone recurrence $(n=1)$ including duplication (data not shown). However, in the OX group, 40 of 
Table III. Characteristics of patients with colon cancers treated with adjuvant chemotherapy following propensity score matching.

\begin{tabular}{|c|c|c|c|}
\hline Characteristic & OX group & PO group & P-value \\
\hline $\mathrm{N}$ & 105 & 105 & \\
\hline Male/female & $50 / 55$ & $49 / 56$ & 0.890 \\
\hline Mean age, years & $62.6(30.0-84.0)$ & $62.9(31.0-89.0)$ & 0.667 \\
\hline Institution (KMMC/KU) & $53 / 52$ & $49 / 56$ & 0.581 \\
\hline Tumor location & & & 0.573 \\
\hline Right & $40(38.1 \%)$ & $44(41.9 \%)$ & \\
\hline Left & $65(61.9 \%)$ & $61(58.1 \%)$ & \\
\hline \multicolumn{4}{|l|}{ Tumor marker } \\
\hline Median CEA & $3.8(0.6-116.0)$ & $2.8(0.5-262.1)$ & 0.088 \\
\hline Median CA19-9 & $9.0(1.5-2351.8)$ & $8.5(1.8-592.2)$ & 0.657 \\
\hline Bowel obstruction & $13(12.4 \%)$ & $11(10.5 \%)$ & 0.664 \\
\hline Surgical procedure & & & 0.818 \\
\hline Laparoscopic & $94(89.5 \%)$ & $95(90.5 \%)$ & \\
\hline Open & $11(10.5 \%)$ & $10(9.5 \%)$ & \\
\hline Median blood loss volume, $\mathrm{ml}$ & $30(2-2965)$ & $30(3-4300)$ & 0.900 \\
\hline Median operating time, $\min$ & $251(88-752)$ & $241(90-778)$ & 0.646 \\
\hline No. of lymph nodes & $25(3-97)$ & $21(2-112)$ & \\
\hline Hospital stay, days & $13(5-137)$ & $13(7-125)$ & 0.738 \\
\hline Postoperative complications & $14(13.3 \%)$ & $15(14.3 \%)$ & 0.842 \\
\hline Tumor differentiation & & & 0.904 \\
\hline Well & $50(47.6 \%)$ & $50(47.6 \%)$ & \\
\hline Moderate & $41(39.1 \%)$ & $43(40.9 \%)$ & \\
\hline Poor & $14(13.3 \%)$ & $12(11.4 \%)$ & \\
\hline T stage & & & 0.468 \\
\hline $\mathrm{T} 1$ & $3(2.9 \%)$ & $7(6.7 \%)$ & \\
\hline $\mathrm{T} 2$ & $11(10.5 \%)$ & $7(6.7 \%)$ & \\
\hline $\mathrm{T} 3$ & $72(68.6 \%)$ & $72(68.6 \%)$ & \\
\hline $\mathrm{T} 4$ & $19(18.1 \%)$ & $19(18.1 \%)$ & \\
\hline $\mathrm{N}$ stage & & & 0.238 \\
\hline N0 & $18(17.1 \%)$ & $28(26.7 \%)$ & \\
\hline N1 & $64(61.0 \%)$ & $58(55.2 \%)$ & \\
\hline $\mathrm{N} 2$ & $23(21.9 \%)$ & $19(18.1 \%)$ & \\
\hline Vessel or lymphatic invasion & $55(52.4 \%)$ & $60(57.1 \%)$ & 0.488 \\
\hline TNM stage & & & $>0.001^{\mathrm{a}}$ \\
\hline II & $14(13.4 \%)$ & $103(29.5 \%)$ & \\
\hline III & $63(86.6 \%)$ & $180(70.5 \%)$ & \\
\hline
\end{tabular}

${ }^{\mathrm{a}} \mathrm{P}<0.05$. CEA, carcinoembryonic antigen; CA, cancer antigen; OX, oxaliplatin; PO, peroral; KMMC, Kitakyushu Municipal Medical Center; KU, Kyushu University Hospital.

$149(26.8 \%)$ patients had CC recurrence, with initial relapse in the liver $(n=12)$ or lung $(n=13)$, tumor dissemination $(n=15)$ and lymph node $(n=10)$, local $(n=5)$ or bone recurrence $(n=1$; data not shown). Patients in the OX group experienced tumor dissemination and local recurrence more frequently than those in the PO group. The 5-year RFS rate was $80.8 \%$ in the PO group and $71.9 \%$ in the OX group (stage IIA, 85.1 and 83.3; IIB, 58.3 and 50.0; IIC, 66.7 and 71.4; IIIA, 93.3 and 100.0; IIIB, 80.5 and 71.3 and IIIC, 45.7 and $61.9 \%$, respectively; data not shown).
Previous studies have shown that OX administration tends to improve RFS (6-8). In order to minimize the impact of any selection bias in comparing the recurrence risk between the two groups and to elucidate the effects of OX for patients with $\mathrm{CC}$, propensity score matching was performed. Patient characteristics following matching are presented in Table III. Before matching, the prognosis of OX group patients was significantly poorer than that of PO group ( $\mathrm{P}=0.0462$; Fig. $2 \mathrm{~A})$, potentially due to strong therapeutic selection bias. After matching, the prognosis of the OX group was better than that of the PO 
A

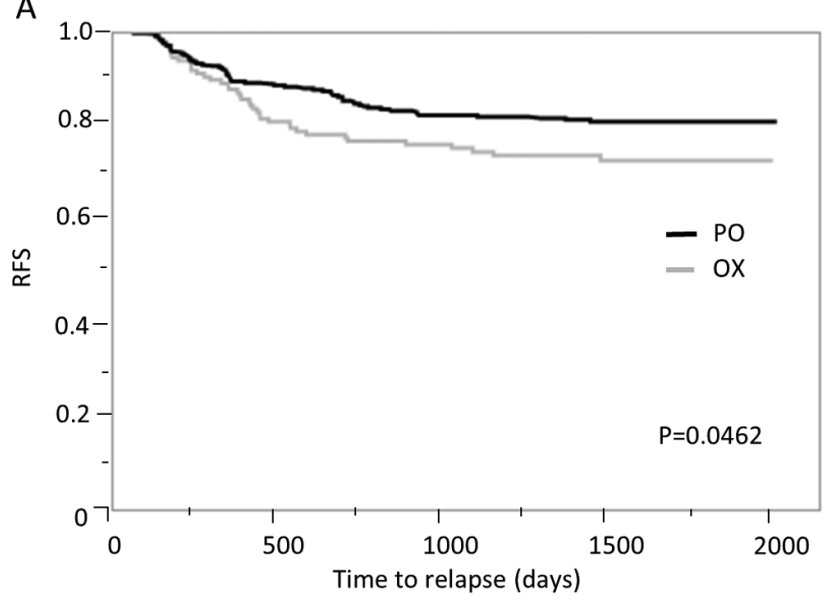

B

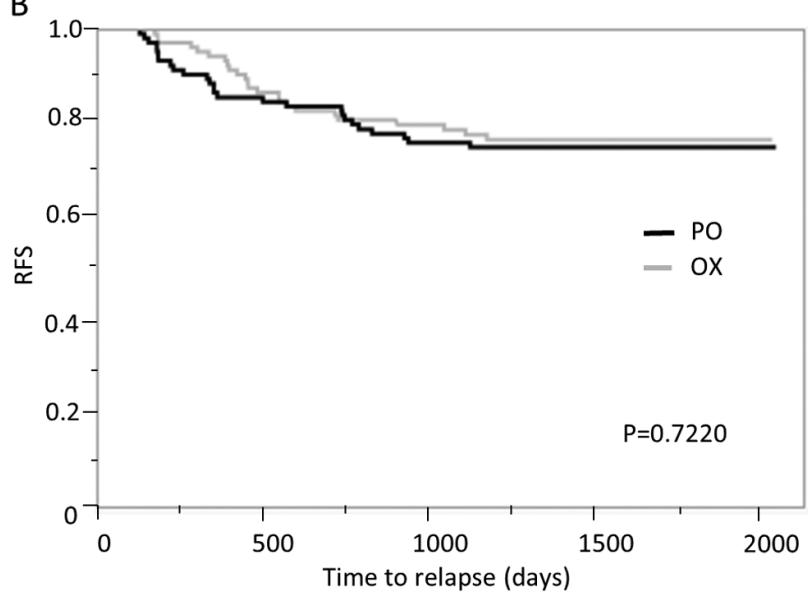

Figure 2. Survival curve of patients with adjuvant chemotherapy before and after propensity matching. (A) Kaplan-Meier survival analysis demonstrated that RFS was poor in the OX group compared with the PO group. (B) Following propensity matching, RFS improved with OX addition (P=0.7220). RFS, recurrence-free survival; OX, oxaliplatin; PO, peroral.

A

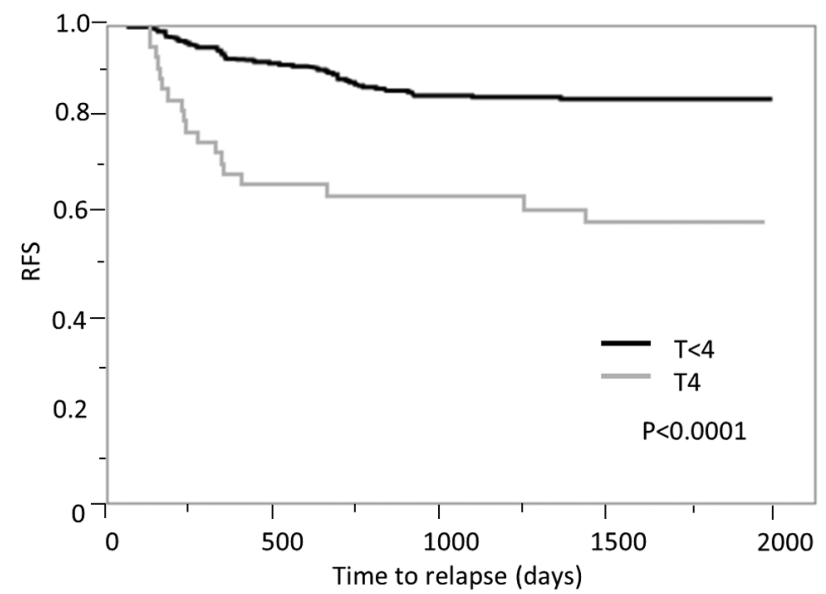

C

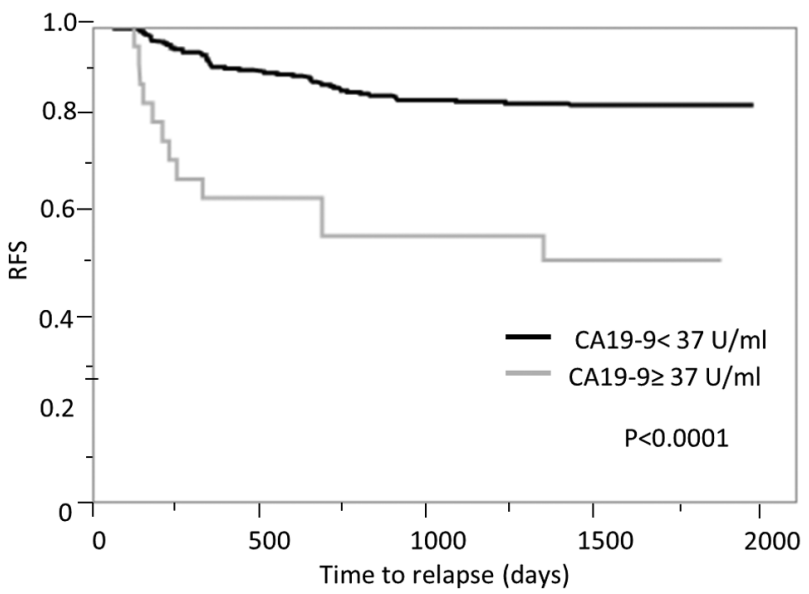

B

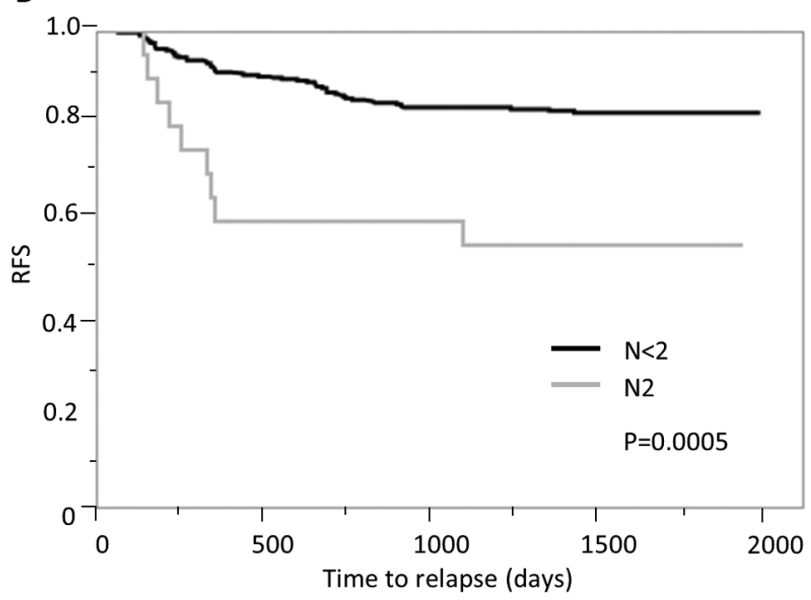

D

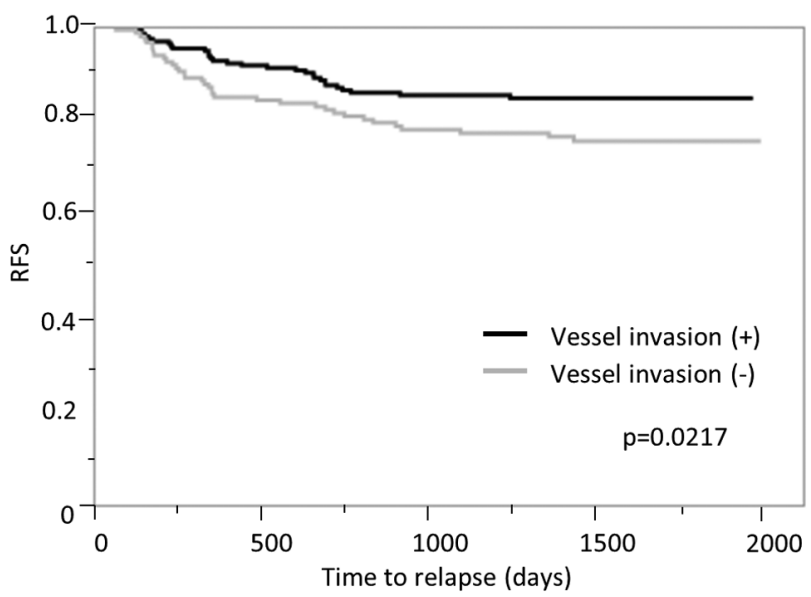

Figure 3. Kaplan-Meier survival analysis showed that (A) T4, (B) N2, (C) high CA19-9 and (D) lymphatic or vessel invasion are poor prognostic factors in RFS . RFS, recurrence-free survival; CA, cancer antigen.

group, although this was not significant $(\mathrm{P}=0.7220$; Fig. 2B), which suggests an additional effect of OX for patients with stage II/III CC.
Risk factors of cancer recurrence for patients with $P O$ regimens. The RFS curve of the PO group is shown in Fig. 3. Univariate analysis showed that T4 $(\mathrm{P}<0.0001$; Fig. 3A), 
Table IV. Univariate and multivariate Cox proportional hazards analysis of predictive factors for cancer relapse in peroral group.

A, Preoperative

\begin{tabular}{lcc}
\hline & Univariate analysis & Multivariate analysis \\
\cline { 2 - 3 } Factor & P-value & Risk ratio (95\% CI) \\
\hline Institution & 0.0824 & \\
Bowel obstruction & 0.8897 & \\
Tumor location & 0.8396 & \\
CEA $>5 \mathrm{ng} / \mathrm{ml}$ & 0.3429 & $3.367(1.773-6.393)$ \\
CA19-9 $>37 \mathrm{U} / \mathrm{ml}$ & $<0.0001^{\mathrm{a}}$ & $0.0002^{\mathrm{a}}$ \\
\hline
\end{tabular}

B, Surgical

\begin{tabular}{lcc}
\hline & Univariate analysis & Multivariate analysis \\
\cline { 2 - 3 } Factor & P-value & Risk ratio (95\% CI) \\
\hline Procedure, laparoscopic vs. open & 0.7307 & \\
Blood loss volume $\geq 300 \mathrm{ml}$ & 0.4415 & \\
Operating time $\geq 300 \mathrm{~min}$ & 0.1515 & \\
Postoperative complications & 0.0800 & \\
\hline
\end{tabular}

C, Histological

\begin{tabular}{lccc}
\hline & Univariate analysis & & Multivariate analysis \\
\cline { 2 - 3 } Factor & P-value & Risk ratio (95\% CI) & \\
\hline Grade & 0.7605 & $2.947(1.698-5.113)$ & $0.0001^{\mathrm{a}}$ \\
T4 vs. T 33 & $<0.0001^{\mathrm{a}}$ & $2.704(1.304-5.609)$ & $0.0075^{\mathrm{a}}$ \\
$\mathrm{N} 2$ vs. $\mathrm{N} \leq 1$ & $0.0005^{\mathrm{a}}$ & $1.675(1.014-2.765)$ & $0.0437^{\mathrm{a}}$ \\
Lymphatic or vessel invasion & $0.0217^{\mathrm{a}}$ & & \\
\hline
\end{tabular}

${ }^{\mathrm{a}} \mathrm{P}<0.05$. CEA, carcinoembryonic antigen; $\mathrm{CA}$, cancer antigen.

N2 ( $\mathrm{P}=0.0005$; Fig. 3B), high CA19-9 ( $\mathrm{P}<0.0001$; Fig. 3C) and lymphatic or vessel invasion $(\mathrm{P}=0.0217$; Fig. 3D) were significantly associated with worse RFS. Multivariate analysis indicated that high CA19-9 [hazard ratio (HR), 3.367; 95\% CI, 1.773-6.393; P=0.0002], T4 (HR, 2.947; 95\% CI, 1.698-5.113; $\mathrm{P}=0.0001)$, N2 (HR, 2.704; 95\% CI, 1.304-5.609; $\mathrm{P}=0.0075)$ and lymphatic or vessel invasion (HR, 1.675; 95\% CI, 1.014-2.765; $\mathrm{P}=0.0437$ ) were independent predictive factors for cancer recurrence (Table IV).

Subgroup analysis of predictive risk factors for cancer relapse in the propensity score-matched cohort. In order to compare the recurrence risk between patients with PO and OX-containing regimens, subgroup analysis was performed using the propensity score-matched cohort to minimize selection bias. Subgroup analysis of patients with T4, N2, high CA19-9 and lymphatic or vessel invasion revealed an additional effect of OX in the high CA19-9 subgroup ( $\mathrm{P}=0.0016$; Fig. 4A). This was not demonstrated in the subgroup with lymphatic or vessel invasion $(\mathrm{P}=0.9722$; Fig. 4B) but there was an association with a prognostic benefit of OX in the T4 and $\mathrm{N} 2$ subgroups $(\mathrm{P}=0.6084$ and $\mathrm{P}=0.2184$, respectively; Fig. 4C and D), which suggests that patients with T4, N2 and high CA19-9 may benefit from OX-containing regimens such as FOLFOX and CapOX.

\section{Discussion}

In Japan, postoperative adjuvant chemotherapy following R0 resection is recommended for patients with high-risk stage II or III CRC by the Japanese Society for Cancer of the Colon and Rectum (JSCCR) guidelines 2019. The recommended regimens include not only OX-containing regimens, such as FOLFOX and CapOX, but also 5-FU/LV, capecitabine, UFT/LV and S-1 (23). At Kitakyushu Municipal Medical Center and Kyushu University Hospital, administration of PO fluorinated pyrimidine-based therapy without OX is common, especially for patients with high-risk stage II without lymph node metastasis, relatively low-risk stage III without T4 and $\geq \mathrm{N} 2$ or high-risk stage III with advanced age or comorbidity 
A

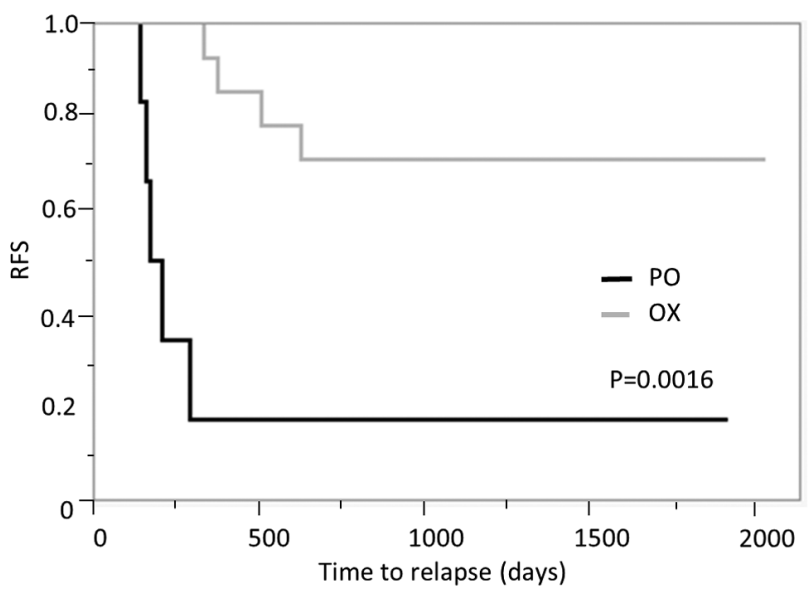

C

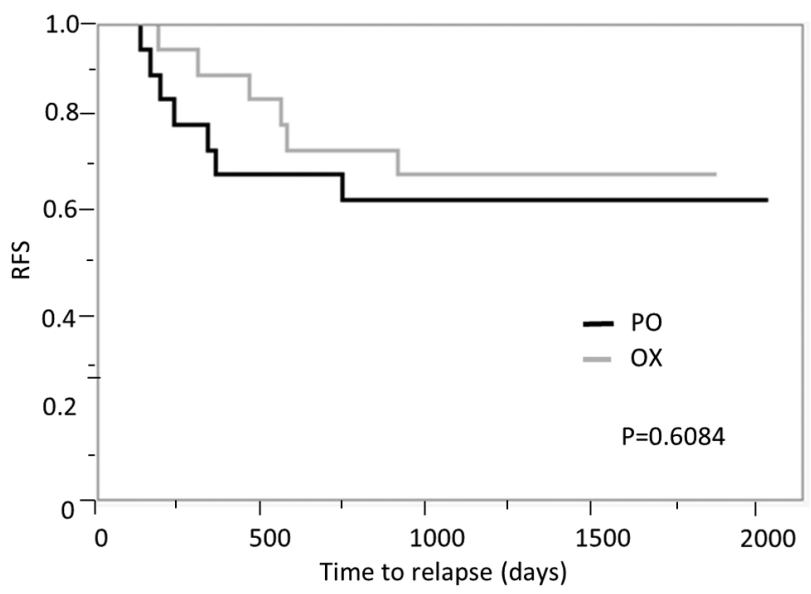

B

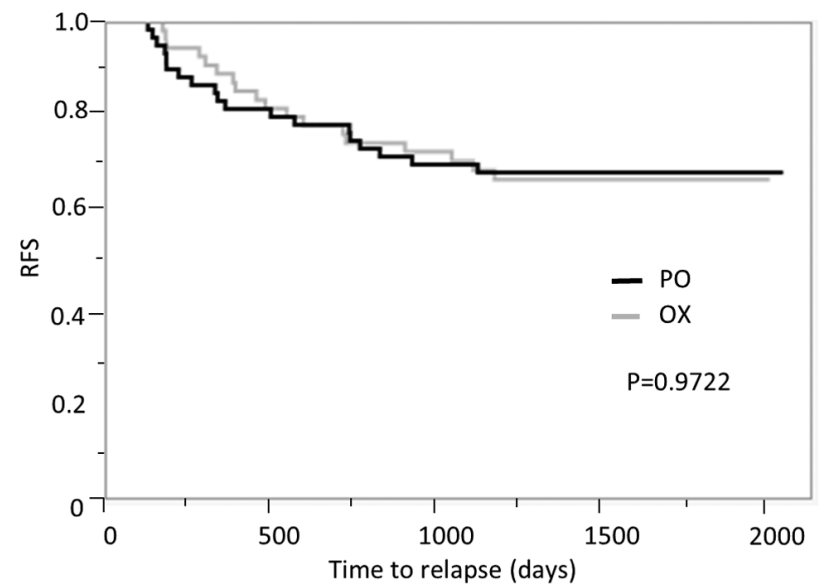

D

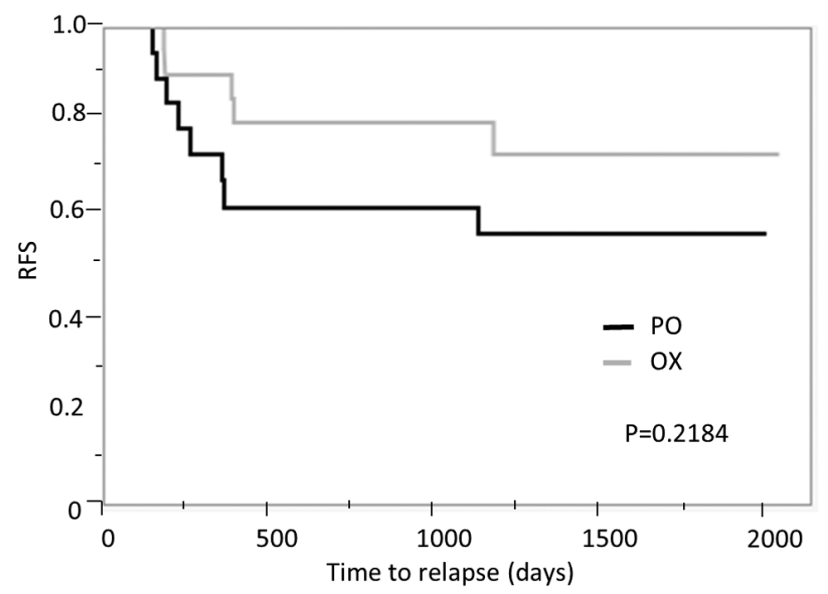

Figure 4. Survival curve of patients with adjuvant chemotherapy following propensity matching. (A) Subgroup analysis following propensity score matching revealed that OX had a significant additional impact on recurrence in the high CA19-9 subgroup. (B) In the vessel or lymphatic invasion-positive subgroup, there was no difference between the PO and OX groups. In the (C) T4 and (D) N2 subgroups, there was no significant difference between the PO and OX groups; both were associated with the prognostic benefit of OX but this was not significant. OX, oxaliplatin; CA, cancer antigen; PO, peroral; RFS, recurrence-free survival.

and patients who prefer to avoid adverse effects, such as persistent peripheral neuropathy. This may reflect the situation in other hospitals in Japan. The stage at which patients with CRC should receive adjuvant chemotherapy is known but it remains controversial as to which patients should be treated with OX-containing regimens.

The present study investigated the predictive factors for recurrence following curative resection in patients with $\mathrm{CC}$ treated with PO adjuvant chemotherapy. Our findings indicated that patients with T4, N2, high CA19-9 and lymphatic or vessel invasion had a high risk of recurrence when treated with PO regimens as postoperative adjuvant chemotherapy. It has been previously reported that T4, N2, high CA19-9 and lymphatic or vessel invasion are poor prognostic factors that affect the survival rate of patients with CC (15-18), but to the best of our knowledge, the present study is the first to demonstrate that these factors are associated with high recurrence rate in patients receiving PO chemotherapy regimens. In Japan, PO regimens are widespread, therefore these results may reflect real-world use of peroral adjuvant chemotherapy for stage II/III CC in Japan.

Previous proportional hazards model survival analyses have identified T4, N2, high tumor marker, postoperative complications and lymphatic or vessel invasion as poor prognostic factors (15-18,24-26). Here, patients in the PO group with high CA19-9, T4, N2, and lymphatic or vessel invasion had a poor prognosis, but high CEA and postoperative complications were not predictive factors for cancer relapse. Previous studies have reported that high expression levels of tumor markers, such as CEA and CA19-9, affect survival in patients with $\mathrm{CC}(16,25,26)$. The present study also indicated that high CA19-9 was an independent predictive marker of recurrence; moreover, high CA19-9 was the only factor to reveal a significant improvement in RFS following OX administration in the propensity score-matched cohort, despite the small number of patients. CEA has also been reported to be a marker of poor prognosis (26), but this was not reflected in the present study and cancer recurrence in patients with high preoperative CEA levels has been observed. The cutoff value of the tumor marker was determined according to the upper limit of normal at Kitakyushu Municipal Medical Center and Kyushu University Hospital to eliminate arbitrariness. Because there is no validated or well-established cutoff for elevated CEA, another appropriate cutoff value for CEA may exist. Postoperative morbidity, such as anastomotic failure, ileus and 
surgical site infection, was not a recurrence risk factor in this study. Adjuvant chemotherapy could not be administered to these frail patients in principle; these patients were assigned to a group without adjuvant chemotherapy and only patients with relatively mild complications began treatment with PO regimens.

Randomized control trials, such as MOSAIC, NSABP C-07 and XELOXA, have shown that the addition of OX to fluorinated pyrimidine-based therapies improves patient outcome in postoperative adjuvant settings (5-8) and OX-containing regimens are recommended as adjuvant chemotherapy for patients with CC by National Comprehensive Cancer Network and JSCCR guidelines $(22,27)$. However, a subgroup analysis of these trials did not demonstrate that OX-containing regimens decrease the risk of cancer recurrence in patients with T4, $\mathrm{N} 2$ and lymphatic or vessel invasion, which suggests that the prognostic benefit of OX is limited in these patients compared with other patients in these previous studies (5-8).

Subgroup analysis before propensity score matching also failed to demonstrate the additional effect of OX in patients with high tumor markers, T4, and lymphatic or vessel invasion. Propensity score matching analysis to adjust clinicopathological factors also showed that OX-containing regimens improved RFS in the high CA19-9 subgroup and that there was an association with RFS improvement in T4 or N2 patients treated with OX-containing regimens compared with $\mathrm{PO}$ regimens. The lack of significance may be due to the small number of patients. These results suggest that OX may confer a benefit to prognosis for patients without these factors.

A limitation of the present study was the retrospective analysis of patients with $\mathrm{CC}$ who were treated at two tertiary medical institutions. In a previous randomized controlled trial, S-1 did not show non-inferiority compared with capecitabine for disease-free survival (28). S-1 was administered prior to this study and there was a potential for selection bias if S-1-treated patients were excluded. Therefore, patients treated with S-1 were included. In addition, the inclusion of patients and physician preferential regimens conferred a selection bias. Strong selection biases can exist when patients without adjuvant chemotherapy are excluded. However, the present study limited patients to those with adjuvant chemotherapy because the aim was to determine predictive factors for cancer recurrence in patients treated with $\mathrm{PO}$ regimens as adjuvant chemotherapy and to investigate the additional effect of $\mathrm{OX}$ in patients with stage II/III CC. Propensity score matching analysis was performed to minimize the effect of other clinicopathological factors influencing survival.

The present study demonstrated that high CA19-9, T4 and lymphatic or vessel invasion were predictive markers for cancer recurrence in patients with $\mathrm{PO}$ regimens. $\mathrm{CC}$ recurrence predictive factors for patients treated with $\mathrm{PO}$ regimens were determined; OX-containing regimens may outcome in patients with T4, N2, and high CA19-9. These data promote $\mathrm{OX}$ as a therapeutic regimen for patients with stage II/III CC.

\section{Acknowledgements}

The authors would like to thank Dr Nikki March from Edanz Group for editing a draft of this manuscript.

\section{Funding}

The present study was supported by the Japan Society for the Promotion of Science Grant-in-Aid for Research Activity Start-up and Young Scientists (grant nos. 19K24016 and 20K17621) and Kanae Foundation.

\section{Availability of data and materials}

The datasets used and/or analyzed during the current study are available from the corresponding author on reasonable request.

\section{Authors' contributions}

YM, YT and MN designed the study. MS, YK and YW analyzed the data. SN, KN and KO performed statistical analysis and data interpretation. ST performed histological diagnoses. YM, YT, MS, YK, SN and TN performed surgery and patient care. $\mathrm{KN}$ and $\mathrm{KO}$ contributed to data acquisition. YM drafted the manuscript. YM and MS confirm the authenticity of all the raw data. All authors read and approved the final manuscript.

\section{Ethics approval and consent to participate}

All procedures conformed to the ethical guidelines of the Japanese Government and the Declaration of Helsinki. The study was approved by the ethics committee of Kitakyushu Municipal Medical Center (approval no. 201801055).

\section{Patient consent for publication}

Not applicable.

\section{Competing interests}

The authors declare that they have no competing interests.

\section{References}

1. Siegel RL, Miller KD and Jemal A: Cancer statistics, 2020. CA Cancer J Clin 70: 7-30, 2020.

2. Japanese National Cancer Center: Cancer Registry and Statistics: Cancer Information Service. http://ganjoho.jp/reg_stat/statistics/dl/index.html\#incidence. Accessed December 2020.

3. Inomata M, Shiroshita H, Uchida $\mathrm{H}$, Bandoh T, Akira S, Yamaguchi S, Kurokawa Y, Seki Y, Eguchi S, Wada N, et al: Current status of endoscopic surgery in Japan: The 14th national survey of endoscopic surgery by the Japan society for endoscopic surgery. Asian J Endosc Surg 13: 7-18, 2020.

4. Survival statistics of Japanese association of Clinical Cancer Centers: Cancer survival rates at Japanese Association of Clinical Cancer Centers. https://kapweb.chiba-cancer-registry. org/usage?lang=en. Accessed December 2020.

5. André T, Boni C, Mounedji-Boudiaf L, Navarro M, Tabernero J, Hickish T, Topham C, Zaninelli M, Clingan P, Bridgewater J, et al: Oxaliplatin, fluorouracil, and leucovorin as adjuvant treatment for colon cancer. N Engl J Med 350: 2343-2351, 2004.

6. André T, Boni C, Navarro M, Tabernero J, Hickish T, Topham C, Bonetti A, Clingan P, Bridgewater J, Rivera F and de Gramont A: Improved overall survival with oxaliplatin, fluorouracil, and leucovorin as adjuvant treatment in stage II or III colon cancer in the MOSAIC trial. J Clin Oncol 27: 3109-3116, 2009.

7. Kuebler JP, Wieand HS, O'Connell MJ, Smith RE, Colangelo LH, Yothers G, Petrelli NJ, Findlay MP, Seay TE, Atkins JN, et al: Oxaliplatin combined with weekly bolus fluorouracil and leucovorin as surgical adjuvant chemotherapy for stage II and III colon cancer: Results from NSABP C-07. J Clin Oncol 25: 2198-2204, 2007. 
8. Haller DG, Tabernero J, Maroun J, de Braud F, Price T, Van Cutsem E, Hill M, Gilberg F, Rittweger K and Schmoll HJ: Capecitabine plus oxaliplatin compared with fluorouracil and folinic acid as adjuvant therapy for stage III colon cancer. J Clin Oncol 29: 1465-1471, 2011.

9. Hoff PM, Saad ED, Costa F, Coutinho AK, Caponero R, Prolla G and Gansl RC: Literature review and practical aspects on the management of oxaliplatin-associated toxicity. Clin Colorectal Cancer 11: 93-100, 2012.

10. Lembersky BC, Wieand HS, Petrelli NJ, O'Connell MJ, Colangelo LH, Smith RE, Seay TE, Giguere JK, Marshall ME, Jacobs AD, et al: Oral uracil and tegafur plus leucovorin compared with intravenous fluorouracil and leucovorin in stage II and III carcinoma of the colon: Results from National Surgical Adjuvant Breast and Bowel Project Protocol C-06. J Clin Oncol 24: 2059-2064, 2006.

11. Shimada Y, Hamaguchi T, Mizusawa J, Saito N, Kanemitsu Y, Takiguchi N, Ohue M, Kato T, Takii Y, Sato T, et al: Randomised phase III trial of adjuvant chemotherapy with oral uracil and tegafur plus leucovorin versus intravenous fluorouracil and levofolinate in patients with stage III colorectal cancer who have undergone Japanese D2/D3 lymph node dissection: Final results of JCOG0205. Eur J Cancer 50: 2231-2240, 2014.

12. Twelves C, Scheithauer W, McKendrick J, Seitz JF, Van Hazel G, Wong A, Díaz-Rubio E, Gilberg F and Cassidy J: Capecitabine versus 5-fluorouracil/folinic acid as adjuvant therapy for stage III colon cancer: Final results from the X-ACT trial with analysis by age and preliminary evidence of a pharmacodynamic marker of efficacy. Ann Oncol 23: 1190-1197, 2012

13. Yoshida M, Ishiguro M, Ikejiri K, Mochizuki I, Nakamoto Y, Kinugasa Y, Takagane A, Endo T, Shinozaki H, Takii Y, et al: ACTS-CC study group. S-1 as adjuvant chemotherapy for stage III colon cancer: A randomized phase III study (ACTS-CC trial). Ann Oncol 25: 1743-1749, 2014

14. Oki E, Murata A, Yoshida K, Maeda K, Ikejiri K, Munemoto Y, Sasaki K, Matsuda C, Kotake M, Suenaga T, et al: A randomized phase III trial comparing S-1 versus UFT as adjuvant chemotherapy for stage II/III rectal cancer (JFMC35-C1: ACTS-RC). Ann Oncol 27: 1266-1272, 2016.

15. Benson AB III, Schrag D, Somerfield MR, Cohen AM, Figueredo AT, Flynn PJ, Krzyzanowska MK, Maroun J, McAllister P, Van Cutsem E, et al: American society of clinical oncology recommendations on adjuvant chemotherapy for stage II colon cancer. J Clin Oncol 22: 3408-3419, 2004.

16. Yu Z, Chen Z, Wu J, Li Z and Wu Y: Prognostic value of pretreatment serum carbohydrate antigen 19-9 level in patients with colorectal cancer: A meta-analysis. PLoS One 12: e0188139, 2017.

17. Betge J, Pollheimer MJ, Lindtner RA, Kornprat P, Schlemmer A, Rehak P, Vieth M, Hoefler G and Langner C: Intramural and extramural vascular invasion in colorectal cancer: Prognostic significance and quality of pathology reporting. Cancer 118 628-638, 2012
18. Akagi Y, Adachi Y, Ohchi T, Kinugasa T and Shirouzu K: Prognostic impact of lymphatic invasion of colorectal cancer: A single-center analysis of 1,616 patients over 24 years. Anticancer Res 33: 2965-2970, 2013.

19. Brierley JD, Gospodarowicz MK and Wittekind C (eds): TNM Classification of Malignant Tumours. 8th edition. John Wiley and Sons, Inc., New York, NY, 2017.

20. Japanese Society for Cancer of the Colon and Rectum: Japanese Classification of Colorectal, Appendiceal and Anal Carcinoma, Third English edition. Kanehara \& Co., Ltd., Tokyo, 2019.

21. Dindo D, Demartines N and Clavien PA: Classification of surgical complications: A new proposal with evaluation in a cohort of 6336 patients and results of a survey. Ann Surg 240: 205-213, 2004.

22. Watanabe Y, Watanabe M, Suehara N, Saimura M, Mizuuchi Y, Nishihara K, Iwashita T and Nakano T: Billroth-I reconstruction using an overlap method in totally laparoscopic distal gastrectomy: Propensity score matched cohort study of short- and long-term outcomes compared with Roux-en-Y reconstruction. Surg Endosc 33: 3990-4002, 2019.

23. Hashiguchi Y, Muro K, Saito Y, Ito Y, Ajioka Y, Hamaguchi T, Hasegawa K, Hotta K, Ishida H, Ishiguro M, et al: Japanese society for cancer of the colon and rectum (JSCCR) guidelines 2019 for the treatment of colorectal cancer. Int J Clin Oncol 25: $1-42,2020$.

24. Burton S, Norman AR, Brown G, Abulafi AM and Swift RI: Predictive poor prognostic factors in colonic carcinoma. Surg Oncol 15: 71-78, 2006.

25. Zhou W, Yang F, Peng J, Wang F, Lin Y, Jiang W, Yang X, Li L, Lu Z, Wan D, et al: High pretreatment serum CA19-9 level predicts a poor prognosis for patients with stage III colon cancer after curative resection and adjuvant chemotherapy. J Cancer 10: 3810-3818, 2019

26. Chapman MA, Buckley D, Henson DB and Armitage NC: Preoperative carcinoembryonic antigen is related to tumour stage and long-term survival in colorectal cancer. Br J Cancer 78: 1346-1349, 1998

27. Benson AB, Venook AP, Al-Hawary MM, Cederquist L, Chen YJ, Ciombor KK, Cohen S, Cooper HS, Deming D, Engstrom PF, et al: NCCN guidelines insights: Colon cancer, version 2.2018. J Natl Compr Canc Netw 16: 359-369, 2018.

28. Hamaguchi T, Shimada Y, Mizusawa J, Kinugasa Y, Kanemitsu Y, Ohue M, Fujii S, Takiguchi N, Yatsuoka T, Takii Y, et al: Capecitabine versus S-1 as adjuvant chemotherapy for patients with stage III colorectal cancer (JCOG0910): An open-label, non-inferiority, randomised, phase 3, multicentre trial. Lancet Gastroenterol Hepatol 3: 47-56, 2018. 